\title{
Effects of vacation from work on health and well-being: Lots of fun, quickly gone
}

\author{
Jessica de Bloom $^{\mathrm{a} *}$ Sabine A.E. Geurts ${ }^{\mathrm{a}}$, Toon W. Taris ${ }^{\mathrm{a}, \mathrm{b}}$, Sabine Sonnentag ${ }^{\mathrm{a}, \mathrm{c}}$, \\ Carolina de Weerth ${ }^{\mathrm{a}}$ and Michiel A.J. Kompier ${ }^{\mathrm{a}}$
}

\begin{abstract}
${ }^{a}$ Department of Work and Organizational Psychology, Behavioural Science Institute, Radboud University Nijmegen, The Netherlands; ${ }^{b}$ Department of Social and Organizational Psychology, University of Utrecht, The Netherlands; ' Department of Work and Organizational Psychology, University of Konstanz, Germany
\end{abstract}

\begin{abstract}
Although vacation from work provides a valuable opportunity for recovery, few studies have met the requirements for assessing its effects. These include taking measurements well ahead of the vacation, during the vacation and at several points in time afterwards. Our study on vacation (after ) effects focused on two related questions: (1) Do health and well being of working individuals improve during a vacation? and (2) How long does a vacation effect last after resumption of work? In a longitudinal study covering seven weeks, 96 Dutch workers reported their health and well being levels two weeks before a winter sports vacation, during vacation and one week, two weeks and four weeks after vacation on seven indicators. Participants' health and well being improved during vacation on five indicators: health status, mood, tension, energy level and satisfaction. However, during the first week of work resumption, health and well being had generally returned to pre vacation levels. In conclusion, a winter sports vacation is associated with improvements in self reported health and well being among working individuals. However, these effects fade out rapidly after work resumption. We propose a framework for future vacation research and suggest investigating the role of vacation type, duration and means to prolong vacation relief.
\end{abstract}

Keywords: work related stress; holiday; vacation; health; well being; recovery; work

\section{Introduction}

Research in the field of occupational health has consistently demonstrated the adverse impact of stress in the workplace on individuals' health and well-being (e.g. Belkic, Landbergis, Schnall, \& Baker, 2004; Ferrie, Westerlund, Virtanen, Vahtera, \& Kivimaki, 2008). This harmful effect is, in part, brought about by physiological stress responses that continue or recur during nonwork time when job stressors are no longer present (e.g. Brosschot, Van Dijk, \& Thayer, 2007; Hjortskov et al., 2004). These prolonged physiological stress responses can be amplified by ruminating thoughts about past and potential future stressors (Geurts \& Sonnentag, 2006) and may disturb the person's homeostatic balance ("allostasis," McEwen, 1998), that is,

*Corresponding author. Email: j.debloom@psych.ru.nl 
the balance between the sympathetic nervous system being dominant during effort expenditure (e.g. in response to stressors) and the parasympathetic nervous system being in control during rest and relaxation (e.g. recovery).

Accordingly, recovery during nonwork time plays a crucial role in protecting employees against the adverse effects of exposure to job stressors. According to Geurts and Sonnentag (2006), the essence of recovery is that

... the psychophysiological systems that were activated during work will return to and stabilize at a baseline level, that is, a level that appears in a situation in which no special demands are made on the individual. (p. 483)

The most influential theories on recovery, Effort-Recovery Theory (Meijman \& Mulder, 1998) and Allostatic Load Theory (McEwen, 1998), share the idea that removal of demands previously put on the individual's psychobiological systems is a prerequisite for recovery to occur.

Recovery after work may occur regularly between workdays (e.g. during evening hours and during weekends) and during longer periods of off-job time such as vacations, constituting meta- and macro-recovery, respectively (Sluiter, FringsDresen, Meijman, \& Van der Beek, 2000). Recent diary studies have revealed that workers often recover insufficiently during regular evening hours and weekends, for instance due to working overtime (Fritz \& Sonnentag, 2005; Van Hooff, Geurts, Kompier, \& Taris, 2007). This day-to-day incomplete recovery constitutes a high risk for serious health impairment in the long term (Van Hooff et al., 2005).

Vacation as a longer and relatively uninterrupted period of absence from work is a prime candidate for helping workers to recover more completely from work. Vacation may contribute to recovery from work through a rather passive mechanism of liberation from demands, as well as through the active engagement in valued and positively experienced free-time activities of one's own choice (e.g. family activities and hobbies).

According to Frederickson's Broaden-and-Build Theory (2001), positive emotions produce flourishing by widening people's thought-action repertoires and by building enduring resources (e.g. intellectual, physical, social and psychological). Positive emotions (e.g. joy, contentment and love) experienced during vacation may not only strengthen the social bond with partners, family members and/or friends, they may also break habitual thought patterns and lead to unusual, creative, fresh ideas to solve long-lasting (job-related) problems. Therefore, a vacation may help to build up enduring personal resources that may function as a buffer for future threats.

In the current study we therefore aim to answer two central research questions:

Question 1: Do the health and well being of working individuals improve during a winter sports vacation (i.e. vacation effect)?

Question 2: Once a vacation effect has occurred, how long does it last after resumption of work (i.e. vacation after effects)?

Although vacation is probably the most powerful prototypical respite occasion for working individuals, as yet surprisingly few researchers have addressed its impact on recovery from work. A recent meta-analysis of vacation research (De Bloom et al., 2009) identified only seven studies that met a set of minimum methodological 
requirements for studying the effects of vacation on health and well-being. The results of these studies suggest that vacation has positive, although weak effects on health and well-being, and that these effects fade out quickly after returning home. However, the evidence is still inconclusive, not only because of the small number of vacation studies but also due to suboptimal research designs often applied (De Bloom et al., 2009). We believe that an adequate study design to investigate the impact of vacation on employees' health and well-being comes down to five major criteria. In the following sections we will discuss each of them in more detail.

\section{A proper pre-vacation baseline}

A number of studies included in the meta-analysis scheduled their pre-vacation measurements shortly before participants went on vacation (De Bloom et al., 2009). However, research showed that the time before a trip can be stressful (DeFrank, Konopaske, \& Ivancevich, 2000). In a similar vein, Westman $(2004,2005)$ stated that pre-vacation activities like planning the vacation, travelling to the vacation destination and coordinating work tasks for the period of absence may also cause pre-vacation stress. Accordingly, it is plausible that measurement occasions immediately before vacation are confounded by either "vacation preparation stress" or working to deadlines before leaving ("working ahead-stress"). But it may also be that vacationers look forward to the vacation, inducing enhanced health and wellbeing. In both cases, it is unreasonable to expect that levels of health and well-being in the week before vacation represent baseline levels of a regular working week. Therefore, in the current study, all comparisons to investigate vacation effects were anchored by a baseline during a regular working week, two weeks before vacation.

\section{An on-vacation measurement occasion}

A concern in some earlier vacation studies regards the absence of health and wellbeing measurements during the vacation period itself (for notable exceptions see Eden, 1990; Fritz \& Sonnentag, 2006; Westman \& Eden, 1997). In most of the earlier vacation studies, pre- and post-vacation measurements were compared, and changed levels of health and well-being were attributed to the unmeasured intervention, that is, the vacation (De Bloom et al., 2009). However, attributing a change in health and well-being to the vacation is a fallacy of the "post hoc ergo propter hoc" type ("after this, therefore because of this") because the sequential occurrence of phenomena does not mean that there is a causal relation between these phenomena (Eden, 2001).

The reason for the dominantly chosen pre-post comparisons to determine a vacation effect is presumably that obtaining data while people are on holiday is difficult (Eden, 2001). Some researchers have even described the logistics of locating people during vacation as "nightmarish" (Eden, 1990, p. 182). Furthermore, respondents might possibly not appreciate being examined during their highly valued holidays (i.e. "holy days"), and traditional research materials like paperpencil questionnaires are hard to use in a vacation setting.

However, investigating a vacation effect by only comparing pre-vacation and post-vacation measurements is inadequate because post-vacation measurements are biased by work resumption and fade-out may already have set in. Every measurement occasion after vacation will therefore reflect an after-effect of vacation and probably 
underestimate the genuine vacation effect. Accordingly, the use of a pre- and postvacation design does not allow us to disentangle vacation- and after-effects and can lead to erroneous conclusions about the effect of vacation on health and well-being. As a consequence, it is essential to obtain information about health and well-being during vacation in order to draw such conclusions. In the current study, we included two on-vacation measurement occasions and defined a "genuine" vacation effect as a significant change in health and well-being levels during vacation compared to prevacation baseline levels.

\section{Multiple post-vacation measurement occasions}

Insufficient attention has been paid to the fade-out process of vacation effects, once they have occurred. As a consequence, it remains largely unknown when fade-out sets in, what its exact course is and when positive after-effects of vacation have completely vanished (De Bloom et al., 2009). Vacation effects are by definition temporary, as any positive effect of vacation will fade out sooner or later, for instance, due to the renewed exposure to work demands. Because previous research suggests that vacation effects fade out rapidly (De Bloom et al., 2009), it is necessary to measure levels of health and well-being immediately after vacation.

In addition, only a few vacation studies have employed more than one postvacation measurement occasion and, if they have done so, the time lag between the two post-vacation occasions has varied widely. In most cases, the first post-vacation occasion has been scheduled in the first week after work resumption and the second post-vacation occasion at least two weeks later (Etzion, 2003; Westman \& Eden, 1997; Westman \& Etzion, 2001). As a result, there has been no information available on health and well-being during the second week after vacation. To close this time gap, we collected data not only in the first week but also in the second work week after vacation.

Occasionally, previous studies have found longer lasting vacation effects (e.g. Westman \& Eden, 1997). Moreover, De Lange and colleagues suggest that longitudinal studies should apply many follow-up measures that are both evenly and unevenly spaced (De Lange, Taris, Kompier, Houtman, \& Bongers, 2003). Therefore, we also included a third post-vacation occasion four weeks after work resumption.

\section{Minimalism and simple comparisons}

Vacation research is complex because it necessarily involves a repeated-measures design. Comparisons between measurement occasions to investigate vacation effects and their duration should be as straightforward, logical and simple as possible. In our view, the essence of vacation research can be reduced to the vacation effect and its potential after-effects.

A vacation effect reflects the difference in health and well-being levels between the pre-vacation measurement occasion (baseline) and the on-vacation measurement occasion. A comparison of the post-vacation measurement occasions with the onvacation measurement occasion reveals whether there may be short-term, mid-term and long-term after-effects of a vacation period. To determine when vacation effects have diminished completely (i.e. baseline levels are attained again) it makes sense to also compare post-vacation measurements with pre-vacation baseline levels. 
Therefore, in our study, after-effects were investigated by comparing health and wellbeing levels after vacation with both on-vacation levels and pre-vacation baseline levels.

\section{Equal and exact timing of measurement occasions for every participant}

While earlier vacation studies had "... no precedent for ideal timing ..." of measurements (Westman \& Eden, 1997, p. 519) and were often rather vague in reporting when exactly measurements took place, we could base the timing of our measurement occasions on earlier findings (see reasoning above) and link every occasion to an identical point in time before, during and after vacation for every single participant. Even the time of the day was kept as constant as possible.

The pre-vacation baseline levels (Pre) were measured two weeks before vacation. The on-vacation levels (Inter) were measured during vacation itself, on the second day after arrival and on the second-last day before departure. The post-vacation levels were measured during the first (Post 1), the second (Post 2) and the fourth week (Post 3) after returning home and resuming work. Figure 1 presents the research design employed in the study.

A vacation effect is present when health and well-being levels during vacation are higher than pre-vacation levels (Pre vs. Inter). The existence of a short-term aftereffect can be detected by comparing the on-vacation measurement occasion with the first post-vacation measurement occasion (Inter vs. Post 1). In case of an improvement in health and well-being from Pre to Inter and no significant differences between Inter and Post 1, vacation effects apparently persist which is supportive of a short-term after-effect.

If post-vacation levels are lower than on-vacation levels, these post-vacation levels will be compared with pre-vacation levels to determine when baseline levels are reached again. In the case of significant differences between the pre-vacation and the first post-vacation measures (Pre vs. Post 1), vacation effects apparently endure (supportive of a short-term after-effect).

The existence of a mid-term after-effect will become evident by comparing the second post-vacation occasion with the on-vacation measurement occasion (Inter vs.

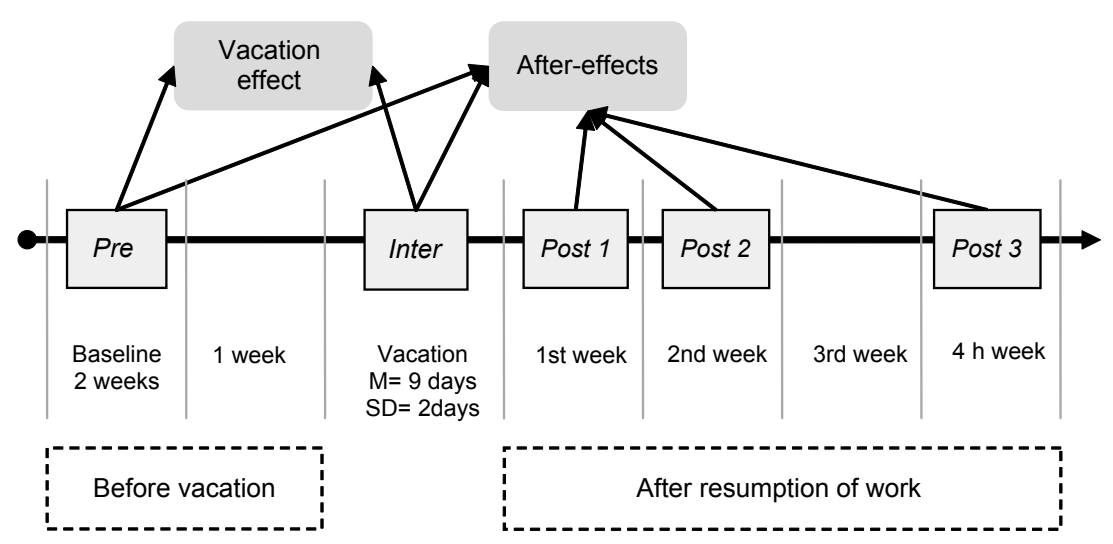

Figure 1. Research design for the current study. 
Post 2) as well as with the pre-vacation levels (Pre vs. Post 2). A significant difference between the pre-vacation and the second post-vacation levels would be supportive of a mid-term after-effect.

If participants' health and well-being levels on the second post-vacation occasion are still higher than baseline levels (indicating that the vacation effect still persists), we proceed with a final set of comparisons (Inter vs. Post 3 and Pre vs. Post 3) to determine if vacation has long-term after-effects.

\section{Method}

\section{Data collection procedure}

We carried out a longitudinal field study on winter sports vacations because this type of holiday normally covers one week and vacationers usually have no more than one or two days off before departure and after return. As a result, vacation duration and the time before and after vacation were roughly comparable for all participants. The same is true for the vacation activities that people typically engage in: winter sport activities during the day (Nordic skiing, alpine skiing, snowboarding, sledding, skating) and socializing (après-ski) in the evening. Consequently, winter sports holidays represent a type of vacation that is more uniform with respect to activities and duration than, for instance, summer vacations and therefore well suited for our research purposes.

Our study covered a time span of seven weeks around the vacation period, including the vacation itself and took place between 15 February and 15 April 2008. On all measurement occasions during working periods that is, two weeks before vacation (Pre), and the first (Post 1), second (Post 2) and fourth week (Post 3) after returning home, the participants received an e-mail with a link to a digital diary twice a week. Participants were asked to complete the diary just before bedtime on a fulltime working day. To make sure that participants would not forget to complete the digital diary in the evening, they additionally received a reminder text message (SMS) on their cell phone earlier that day.

In order to take on-vacation measures of health and well-being, the participants were provided with cell phones with international pre-paid SIM cards to take with them on holiday. They were asked to return the cell phones after returning home in a pre-stamped envelope. While on holiday, every participant was called on this cell phone and interviewed by one of the researchers on the second day after arrival and on the second-last day before departure between 5 and 7 pm (Inter measurement occasion).

Before the cycle of data collection started, participants received a card with an overview of their personal measurement occasions during the seven-week period. After the whole cycle of data collection, respondents were thanked for their participation, were given the opportunity to comment on the research procedure and received information about the time when the results were expected to be published in the academic literature and on our website.

To encourage participation and to reduce missing data, we announced a lottery prize among all participants: a one-week winter sports holiday for the next winter sports season. Chances of winning were higher for participants who returned all questionnaires than for participants who missed measurement occasions. In May, 
the winner was drawn by lot and made public. Moreover, every participant received 10 Euro as pre-paid talk credit on his or her vacation phone.

\section{Missing data: prevention and treatment}

Missing data constitute a major problem in longitudinal designs (Taris, 2000) and effective strategies to prevent and deal with missing data were applied. First of all, because we assumed that especially well-informed participants would comply with our intensive data collection procedure, we devoted much attention to instructing them on the research procedure.

Second, we scheduled two measurement occasions within each week. In order to obtain a reliable indicator of the week-level of health and well-being, the two withinweek measures of a particular health and well-being indicator were averaged. This approach also served to prevent missing data in case of a single non-answered prompt during a workweek. In that case the other measurement in that week (if available) was treated as the week average.

Third, for data collection, we used electronic mail and SMS to remind the participants to fill in the questionnaires at the correct moment in time. Because we used digital diaries, we could recognize un-answered prompts immediately, and a detailed non-completion script was applied for the digital diaries as well as the telephone surveys. These strategies also reduced the amount of missing data.

Finally, in anticipation of possible technical problems with the mobile vacation phones, a sealed envelope containing a paper-and-pencil questionnaire with the interview questions was sent to the participants before departure as backup. When all attempts to reach a participant by phone failed, we sent an SMS that allowed participants to open the envelope and to fill in the questionnaire. Nine measurements during vacation were in fact paper-and-pencil questionnaires returned in a prestamped envelope.

In order to guarantee the reliability and comparability of the measurements, we excluded data from the digital diary (a) when participants filled in the questionnaire on nonwork days instead of on fulltime working days as requested, and (b) when participants completed the questionnaire between 6 am and $6 \mathrm{pm}$ instead of just before bedtime as requested.

Considering the 10 measurements per individual, 83 respondents replied to at least eight single measurements (digital diaries and telephone interviews during vacation). Based on a maximum of 960 possible single measurements in this study (10 measurements in 96 persons), the overall completion rate was $87 \%$ (834 measurements). The combination of the 10 measurements (two measurements a week) into five occasions resulted in even more reliable week-indicators and high completion rates: $100 \%(N=96)$ on Pre, 98\% $(N=94)$ on Inter, $90 \%(N=86)$ on Post 1 and $96 \%(N=92)$ on Post 2 and Post 3 . For 83 of the 96 participants data sets were complete (no missing data on any of the five occasions).

\section{Participants}

To recruit participants in the Netherlands, we distributed information via travel agencies, winter sports websites, shops for skiing-equipment, winter sports journals and newspaper ads. Additionally, we visited a winter sports fair and contacted 
ski-clubs (i.e. sporting clubs for skiers who jointly exercise for their next winter sports holiday).

As a result of the recruitment procedure, 176 persons indicated that they were interested in taking part in this study. After administering detailed information about the research procedure and promising confidentiality, these 176 persons received a phone call from one of the researchers. During this call, possible questions about the research scheme were answered and the participants were screened for participation prerequisites: participants (i) had to work at least 24 hours per week (18 exclusions), (ii) go on winter sports vacation for at least one week between 15 February and 15 April 2008 (22 exclusions), and (iii) enrol in the study on time (17 exclusions). Persons working extremely irregular schedules were also excluded (four exclusions). Moreover, a small number of interested persons did not want to be called during vacation (four exclusions), did not use electronic mail (five exclusions) or found the research procedure too burdensome (three exclusions). Another seven persons were excluded because they did not go on vacation after all due to sickness. All in all, of the 176 people who were initially interested, 108 met the inclusion criteria. Of those 108, 96 actually took part in the study, resulting in an $89 \%$ response rate.

The majority of this Dutch sample was male (65\%), the mean age was 44 years $(S D=10$ years) and as regards education $5 \%$ of the sample was lower (no secondary education, lower secondary or junior secondary education), $40 \%$ medium (senior general secondary and university preparation education) and 55\% highly educated (higher professional and higher education). A majority of the respondents were employed $(82 \%)$ while $18 \%$ were self-employed. The participants worked in a variety of sectors: $23 \%$ worked in the commercial sector, $20 \%$ were higher educated specialists (e.g. engineers, ICT-workers), 14\% worked in the service sector, $12 \%$ in health care, $11 \%$ were administrative employees, $7 \%$ were craftsmen or worked in the production industry, $4 \%$ were teachers, and the remaining $9 \%$ worked in other sectors.

The participants worked in general 38 hours per week on average ( $S D=8$ hours), at least 24 contractual hours per week and the total number of weekly work hours (including overtime) varied from 24 to 60 hours. Forty-seven percent of the participants supervised other persons whereas 53\% had no supervisory tasks. In terms of their personal living situation, the majority of the respondents $(57 \%)$ was married and lived with at least one child, 29\% were married and lived without children, $9 \%$ were unmarried and lived alone, $2 \%$ were single parents and $2 \%$ lived in their parents' house.

The mean vacation duration was nine days ( $S D=2$ days, range: 7-19 days). Vacation destinations were typical winter sports areas, with the top-three destinations being Austria (70\%), France (15\%) and Switzerland (6\%). Most of the respondents were experienced skiers: every participant had been on a skiing vacation at least one time before, and the average number of previous skiing vacations was $22(S D=15$ times $)$.

\section{Measures}

In order to be able to give a detailed account of health and well-being, we incorporated a range of different health and well-being indicators (H\&W indicators). To prevent non-response we minimized the effort required from the participants and 
maximized user-friendliness by reducing the number of digital diary questions as much as possible. Therefore, we employed seven single-item measures to tap the seven main indicators of health and well-being: sleep quality, health status, mood, fatigue, tension, energy level and satisfaction.

Single-item measures often have a high face validity, and participants value their directness and lack of redundant and repeated comparable items. Accordingly, multiple item measures may be validly replaced by single-item measures and still be psychometrically acceptable if the underlying constructs are sufficiently onedimensional and unambiguous to the participants (e.g. Elo, Leppänen, \& Jahkola, 2003; Van Hooff, Geurts, Taris, \& Kompier, 2007).

For simplicity, we adapted response scales based on the well-known basic Dutch grade notation system ranging from 1 (extremely low/negative) to 10 (extremely high/ positive) and anchored the first and the last grade. The exact wording of each singleitem measure and the anchors can be found in Table 1.

\section{Statistical approach}

The data were analyzed in a 5 (Occasion: five occasions) $\times 7$ (health and well-being: seven H\&W indicators) multivariate analysis of variance (MANOVA) with repeated measures on both Occasion (the independent variable or factor) and $\mathrm{H} \& \mathrm{~W}$ (our criterion variables). Subsequently, follow-up univariate ANOVAs were performed for each of the seven H\&W indicators separately (cf. DeShon \& Morris, 2003).

The vacation effect (Question 1) was examined by computing Fisher's Least Significant Difference (LSD) test for Pre versus Inter, presenting Cohen's $d$ for paired observations (Cohen, 1988, p. 46) as an effect size. Following Cohen (1988) we distinguished among small ( 0 to 0.5$)$, medium $(0.5$ to 0.8$)$ and large $(>0.8)$ effect sizes.

In order to test if there was a short-term after-effect of the vacation (Question 2), we compared the on-vacation measure (Inter) with the first post-vacation occasion (Post 1). In the next step, the comparison of Pre versus Post 1 told us if H\&W indicators had returned to baseline levels.

For H\&W indicators that did not attain baseline at Post 1, we examined post-hoc Fisher's LSD differences between Inter and Post 2 to test if vacation effects still

Table 1. Description of the seven single item measures used in this study.

\begin{tabular}{|c|c|c|c|}
\hline $\begin{array}{l}\text { Health and } \\
\text { well being } \\
\text { indicators }\end{array}$ & Single item measure & $\begin{array}{l}\text { A score of } \\
1 \text { means... }\end{array}$ & $\begin{array}{l}\text { A score of } \\
10 \text { means... }\end{array}$ \\
\hline Sleep quality & How did you sleep last night? & Very badly & Very good \\
\hline Health status & How healthy did you feel today? & Very unhealthy & Very healthy \\
\hline Mood & How was your mood today? & Very bad & Very good \\
\hline Fatigue & How fatigued did you feel today? & Not fatigued at all & Very fatigued \\
\hline Tension & How tense did you feel today? & Very calm & Very tense \\
\hline Energy level & $\begin{array}{l}\text { How energetic do you } \\
\text { currently feel? }\end{array}$ & $\begin{array}{l}\text { Absolutely not } \\
\text { energetic }\end{array}$ & Very energetic \\
\hline Satisfaction & $\begin{array}{l}\text { How satisfied do you feel about } \\
\text { this day? }\end{array}$ & $\begin{array}{l}\text { Absolutely } \\
\text { not satisfied }\end{array}$ & Very satisfied \\
\hline
\end{tabular}


persisted and a mid-term after-effect applied. The post-hoc Fisher's LSD test between Pre and Post 2 informed us about the strength and duration of this potential midterm after-effect.

Only in case of a mid-term after-effect, we examined the post-hoc differences between Inter versus Post 3 and Pre versus Post 3 to determine if there was a longterm after-effect.

\section{Results}

\section{Preliminary analysis: descriptive statistics}

Pearson product moment correlations were examined to determine the relationship between the seven different $\mathrm{H} \& \mathrm{~W}$ indicators on the five measurement occasions. The full 35 by 35 table (five occasions multiplied by seven $\mathrm{H} \& \mathrm{~W}$ indicators) is available on request from the first author.

Autocorrelations that can be interpreted as test-retest reliability coefficients ranged from .06, $n s$, for the Pre and Inter measures of sleep, to .67, $p<.001$, for the Post 2 and Post 3 measures of energy level. The correlations among the seven H\&W indicators on the same measurement occasions ranged, for Pre, between -.28 ( $p<.01$, fatigue and sleep quality) and .78 ( $p<.001, \operatorname{mood}$ and satisfaction), for Inter between .08 ( $n s$, satisfaction and energy level) and $.68(p<.001$, mood and health status), for Post 1 between .04 ( $n s$, energy level and sleep quality) and .76 ( $p<.001$, satisfaction and mood), for Post 2 between .09 ( $n s$, energy level and health status) and .82 ( $p<.001$, satisfaction and mood), and for Post 3 between -.16 ( $n s$, tension and sleep quality) and $.71(p<.001$, satisfaction and mood). So, the H\&W indicators were interrelated, but not identical. Mean scores for the seven H\&W indicators across the five measurement occasions are presented in Table 2 and Figure 2.

With regard to the on-vacation measurements of health and well-being, there were no systematic differences between reports collected by telephone interviews and the nine reports collected by paper-and-pencil questionnaires $(t(85)<1.30$, $p>.05)$.

\section{Multivariate analysis}

Multivariate analysis of variance revealed main effects of Occasion, $F(4,79)=7.29$, $p<.001$, and of $\mathrm{H} \& \mathrm{~W}, F(6,77)=140.35, p<.001$, as well as a significant Occasion $\times \mathrm{H} \& \mathrm{~W}$ interaction effect, $F(24,59)=7.20, p<.001$. Hence, health and well-being varied significantly across the five occasions, and this across-time change was different for the various $\mathrm{H} \& \mathrm{~W}$ indicators.

\section{Univariate analysis}

Follow-up univariate ANOVAs for the H\&W indicators across the five measurement occasions revealed that the levels of six indicators varied significantly across the five occasions (Table 2). Sleep quality was the only indicator that did not show an overall occasion effect, $F(4,79)=1.93, n s$, meaning that sleep quality did not differ significantly before, during and after the vacation period. 
Table 2. Means and standard deviations on all five occasions and occasion effects, vacation effects (research question 1) and vacation after effects (research question 2) for all health and well being indicators (H\&W indicators).

\begin{tabular}{|c|c|c|c|c|c|c|c|c|c|c|c|}
\hline \multirow{3}{*}{$\begin{array}{l}\text { Health and } \\
\text { well being } \\
\text { indicators }\end{array}$} & \multirow{2}{*}{\multicolumn{5}{|c|}{ Means and (standard deviations) for five occasions }} & \multirow{3}{*}{$\begin{array}{c}\begin{array}{c}\text { Occasion } \\
\text { effect }\end{array} \\
\begin{array}{c}F \text { partial } \\
\text { eta }\end{array}\end{array}$} & \multicolumn{5}{|c|}{ Effect sizes (Cohen's $d$ ) for various comparisons } \\
\hline & & & & & & & \multirow{2}{*}{$\begin{array}{c}\begin{array}{c}\text { Vacation } \\
\text { effect }\end{array} \\
\begin{array}{c}\text { Pre vs. } \\
\text { Inter }\end{array}\end{array}$} & \multicolumn{2}{|c|}{$\begin{array}{l}\text { Short term } \\
\text { after effect }\end{array}$} & \multicolumn{2}{|c|}{$\begin{array}{l}\text { Mid term } \\
\text { after effect }\end{array}$} \\
\hline & Pre & Inter & Post 1 & Post 2 & Post 3 & & & $\begin{array}{l}\text { Inter vs. } \\
\text { Post } 1\end{array}$ & $\begin{array}{l}\text { Pre vs. } \\
\text { Post } 1\end{array}$ & $\begin{array}{l}\text { Inter vs. } \\
\text { Post } 2\end{array}$ & $\begin{array}{l}\text { Pre vs. } \\
\text { Post } 2\end{array}$ \\
\hline Sleep quality & $\begin{array}{c}7.42 \\
(1.05)\end{array}$ & $\begin{array}{c}7.46 \\
(1.31)\end{array}$ & $\begin{array}{c}7.62 \\
(1.00)\end{array}$ & $\begin{array}{c}7.42 \\
(1.24)\end{array}$ & $\begin{array}{c}7.18 \\
(1.26)\end{array}$ & $\begin{array}{l}1.93 \\
0.09\end{array}$ & - & - & - & - & - \\
\hline Health status & $\begin{array}{c}7.53 \\
(1.24)\end{array}$ & $\begin{array}{c}7.98 \\
(1.26)\end{array}$ & $\begin{array}{l}7.63 \\
(1.44)\end{array}$ & $\begin{array}{c}7.53 \\
(1.12)\end{array}$ & $\begin{array}{c}7.45 \\
(1.04)\end{array}$ & $\begin{array}{l}3.88^{* *} \\
0.16\end{array}$ & $0.40 * *$ & $-0.33^{*}$ & 0.08 & \multirow{2}{*}{\multicolumn{2}{|c|}{$\begin{array}{l}\text { Back to baseline at } \\
\text { Post } 1 \\
\text { Back to baseline at } \\
\text { Post } 1\end{array}$}} \\
\hline Mood & $\begin{array}{c}7.28 \\
(1.17)\end{array}$ & $\begin{array}{c}8.27 \\
(0.99)\end{array}$ & $\begin{array}{c}7.41 \\
(1.10)\end{array}$ & $\begin{array}{c}7.28 \\
(1.13)\end{array}$ & $\begin{array}{c}7.31 \\
(1.12)\end{array}$ & $\begin{array}{l}15.91^{* *} \\
0.45\end{array}$ & $1.01^{* *}$ & $-0.94^{* *}$ & 0.16 & & \\
\hline Fatigue & $\begin{array}{c}4.42 \\
(1.99)\end{array}$ & $\begin{array}{c}4.64 \\
(1.99)\end{array}$ & $\begin{array}{c}3.81 \\
(1.72)\end{array}$ & $\begin{array}{c}4.28 \\
(1.62)\end{array}$ & $\begin{array}{c}4.60 \\
(1.71)\end{array}$ & $\begin{array}{l}3.78 * * \\
0.16\end{array}$ & -0.13 & $0.56^{* *}$ & $0.41 * *$ & 0.22 & 0.10 \\
\hline Tension & $\begin{array}{c}3.43 \\
(1.80)\end{array}$ & $\begin{array}{c}2.31 \\
(1.16)\end{array}$ & $\begin{array}{l}3.25 \\
(1.53)\end{array}$ & $\begin{array}{c}3.55 \\
(1.78)\end{array}$ & $\begin{array}{c}3.71 \\
(1.71)\end{array}$ & $\begin{array}{l}21.68^{* *} \\
0.52\end{array}$ & $1.01 * *$ & $-0.90 * *$ & 0.15 & \multirow{2}{*}{\multicolumn{2}{|c|}{$\begin{array}{l}\text { Back to baseline at } \\
\text { Post } 1 \\
\text { Back to baseline at } \\
\text { Post } 1\end{array}$}} \\
\hline Energy level & $\begin{array}{c}5.90 \\
(1.90)\end{array}$ & $\begin{array}{c}6.84 \\
(1.37)\end{array}$ & $\begin{array}{c}5.96 \\
(1.89)\end{array}$ & $\begin{array}{c}5.90 \\
(1.74)\end{array}$ & $\begin{array}{c}5.51 \\
(1.74)\end{array}$ & $\begin{array}{l}9.13^{* *} \\
0.32\end{array}$ & $0.65 * *$ & $-0.65^{* *}$ & 0.04 & & \\
\hline Satisfaction & $\begin{array}{c}7.32 \\
(1.05)\end{array}$ & $\begin{array}{c}8.14 \\
(1.10)\end{array}$ & $\begin{array}{c}7.34 \\
(1.15)\end{array}$ & $\begin{array}{l}7.25 \\
(1.09)\end{array}$ & $\begin{array}{l}7.28 \\
(0.95)\end{array}$ & $\begin{array}{l}11.43^{* *} \\
0.37\end{array}$ & $0.83^{* *}$ & $-0.84 * *$ & 0.03 & \multicolumn{2}{|c|}{$\begin{array}{l}\text { Back to baseline at } \\
\text { Post } 1\end{array}$} \\
\hline
\end{tabular}

Note: $* p<.05, * * p<.01$. Pre $=$ two weeks before vacation, Inter $=$ during vacation, Post $1=$ first week of work resumption, Post $2=$ second week of work resumption, Post 3 = fourth week of work resumption. Baseline = levels of health and well being indicators two weeks before vacation (Pre). 


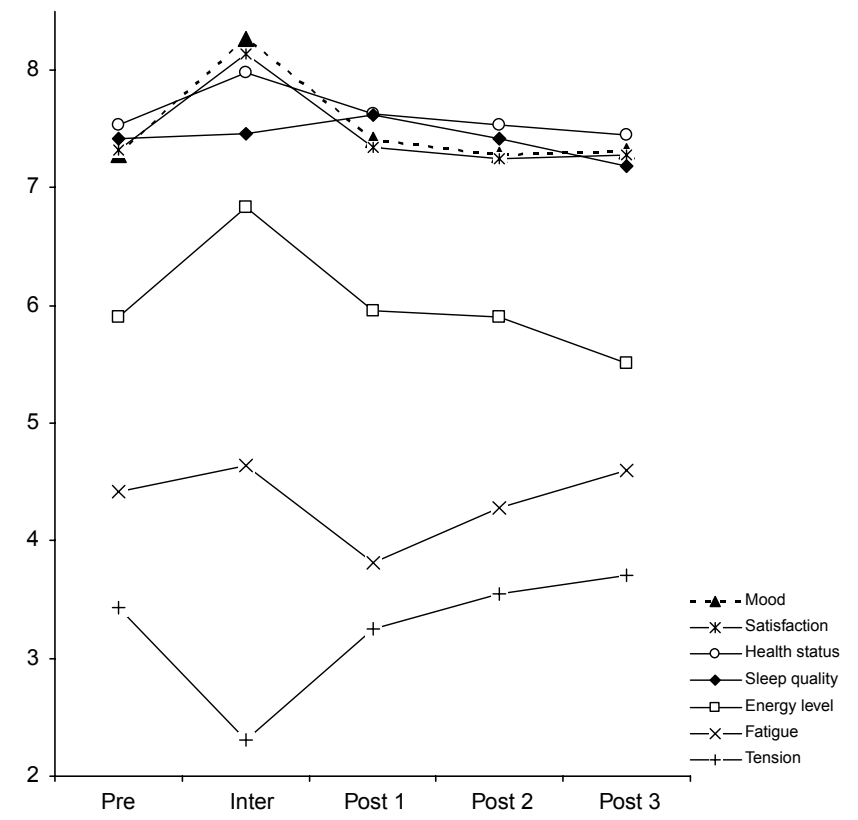

Figure 2. Line diagram of means for health and well being indicators across the five measurement occasions.

\section{Research question 1: Do health and well-being of working individuals improve during a winter sports vacation (i.e. vacation effect)?}

To answer the first research question, we compared the pre-vacation measures of the six $\mathrm{H} \& \mathrm{~W}$ indicators with the measures taken during vacation (Inter). Five out of seven indicators showed an overall occasion effect with Pre levels of health and wellbeing being significantly different from Inter levels $(p<.01)$. During the vacation, participants felt healthier, were in a better mood, felt more energized, were more satisfied and reported lower tension than during the regular working week before they went on vacation. Effect sizes were large for satisfaction $(d=0.83)$, mood $(d=1.01)$ and tension $(d=1.01)$, medium for energy level $(d=0.65)$, and small for health status $(d=0.40)$. The level of fatigue was not significantly different during the vacation period compared to the pre-vacation baseline $(p=.74)$.

Overall, self-reported health and well-being significantly improved during vacation. The mean absolute effect size $d$ for the difference between Pre and Inter in all seven $\mathrm{H} \& \mathrm{~W}$ indicators was 0.54 , indicating a medium-sized positive vacation effect ( $d$ s were 0.03 for sleep, 0.40 for health status, 1.01 for mood, -0.13 for fatigue, 1.01 for tension, 0.65 for energy level, and 0.83 for satisfaction).

Research question 2: Once a vacation effect has occurred, how long does it last after work resumption (i.e. vacation after-effects)?

To test if there was a short-term after-effect, we conducted post-hoc Fisher's Least Significant Difference (LSD) tests for the difference between the on-vacation measure (Inter) and the first post-vacation occasion (Post 1). For all six H\&W 
indicators, there was a significant difference between Inter and Post 1. For five of the six indicators, self-reported health and well-being had declined significantly immediately after participants had returned home and resumed work. Effect sizes were small for health status $(d=-0.33)$, medium for energy level $(d=-0.65)$ and large for satisfaction $(d=-0.84)$, tension $(d=-0.90)$ and mood $(d=-0.94)$. For fatigue, findings were different: levels of fatigue had decreased rather than increased directly after vacation $(d=0.56)$, indicating a positive short-term after-effect.

An inspection of the means of the $\mathrm{H} \& \mathrm{~W}$ indicators (Table 2) already provided interesting insights: an increase from Pre to Inter was followed by an immediate decrease in health and well-being of nearly the same amount from Inter to Post 1 , resulting in almost baseline levels again. The mean score for health status increased by 0.45 points during vacation and decreased by 0.35 points from Inter to Post 1 . The same pattern could be observed for mood (0.99 increase during vacation, 0.86 decrease at Post 1$)$, energy level ( 0.94 increase, 0.88 decrease), and satisfaction (0.82 increase, 0.80 decrease). Tension showed a similar pattern in the reversed direction (1.12 decrease during vacation, 0.94 increase at Post 1). Standardized effect sizes $d$, which enabled us to compare the rise and fall within the seven $\mathrm{H} \& \mathrm{~W}$ indicators relative to each other, mirrored this development across time.

Post-hoc tests of the difference between Pre and Post 1 were non-significant in five of the six $H \& W$ indicators, indicating that during the first week after vacation, there was a return to baseline levels for health status, mood, tension, energy level and satisfaction. The lowest levels of fatigue were found at Post 1 and accordingly there was a significant decrease in fatigue from Pre to Post 1 , resulting in a positive effect size $d$ of 0.41 .

Because every single $\mathrm{H} \& \mathrm{~W}$ indicator except fatigue had reached baseline levels again at Post 1, we only conducted post-hoc tests for a mid-term after-effect in fatigue. As fatigue was lowest on Post 1 and had similar levels at Pre, Post 2 and Post 3 , the differences between Inter versus Post 2 and Pre versus Post 2 were indeed nonsignificant ( $p$ s were .30 and .44 , respectively). So, fatigue had returned to baseline levels at Post 2.

In conclusion, self-reported health and well-being had declined rapidly after resumption of work: five of the six $\mathrm{H} \& \mathrm{~W}$ indicators (health status, mood, tension, energy level, satisfaction) had returned to baseline levels within the first week of work resumption (Post 1), meaning that vacation had no short-term, mid-term or longterm after-effect. Fatigue showed a different pattern of rise and fall, with the lowest level at Post 1 and levels comparable to baseline at Post 2, suggesting a short-term after-effect.

\section{Process evaluation}

In an evaluation of the research procedure, $63 \%$ of the respondents reported to have enjoyed participating in our study and only $17 \%$ found the research procedure a little boring or time consuming. The great majority appreciated the digital diaries (94\%) and $66 \%$ found the reminder SMS very useful. Only a small percentage $(9 \%)$ indicated that the phone call interfered somewhat with their vacation but the great majority $(65 \%)$ indicated that being called during vacation was "no problem." The majority $(93 \%)$ even judged the vacation phones as a very good and creative idea. 


\section{Discussion}

\section{Vacation effect}

Our study provided evidence for improvements in self-reported health and well-being during a winter sport vacation. The average effect size for the vacation effect computed across the seven health and well-being indicators was $d=0.54$ (medium). This effect was present for five of the seven health and well-being indicators employed in this study. In particular, workers felt more satisfied and experienced more positive mood and less tension during vacation compared to a regular prevacation working week. In addition, although to a lesser extent, workers felt more energized and healthier during vacation than before vacation.

These findings strongly support the idea of a vacation as a powerful opportunity to recover from work demands and to benefit from positive free-time experiences. Regarding fatigue and sleep quality, participants' reports did not differ between the on-vacation and the pre-vacation occasions. The finding that mood, tension and satisfaction were more strongly affected by vacation than, for instance, health status may reflect the fact that the former aspects of health and well-being are more sensitive to changes in stressors and work demands and fluctuate more easily from day to day, than the latter.

We believe that current study has several strengths, specifically, a research design with multiple repeated measures pre-, inter- and post-vacation. We succeeded in carrying out 10 repeated measurements per individual (two measurements for each of the five occasions) during a seven-week period in a substantial group of 96 vacationers. Hereby, we applied a proper pre-vacation baseline measurement during a regular working week two weeks prior to vacation and we were able to assess the after-effects of vacation by monitoring health and well-being on three measurement occasions after vacation. Additionally, our study is one of the few studies that measured health and well-being during vacation itself. The importance of the inclusion of on-vacation measurements to determine the "genuine" vacation effect can easily be illustrated: if we had left out the on-vacation occasion, we would falsely have concluded that vacation generally had no positive effect on health and well-being.

The combination of traditional and new media gave us the opportunity to generate rich data sets in a reliable, user-friendly way and to reduce missing data and attrition drastically by acting upon the principle "the more you measure, the less the pleasure." This means, we measured frequently but in a comfortable manner by restricting the number of questions to a minimum and by designing easy-to-use instruments and resources like digital diaries, telephone surveys and SMS reminders. The process evaluation of the participants confirms that our approach was generally experienced positively.

Our findings showed that sleep quality and fatigue had not improved on-vacation compared to the pre-vacation baseline. Previous research has suggested, however, that sleep quality and stress are closely related (e.g. Akerstedt, 2006) and that sleep quality improves in times of low stress (Dahlgren, Kecklund \& Akerstedt, 2005). It is possible that the potential beneficial effects of low stress and rest on sleep quality may have been outweighed by specific vacation circumstances, such as a reduced number of hours sleep, an unfamiliar sleeping environment (e.g. a different bed, different sounds, and light and temperature conditions) and changes in sleep-relevant 
behaviour. Regarding the latter, it is not uncommon during a winter sports vacation to drink substantial amounts of alcohol during the après ski (Meyers, Perrine, \& Caetano, 1997), which might in turn lead to sleep disruption (Roehrs \& Roth, 2001). It is conceivable that the beneficial effect of low stress and rest on sleep quality only occurs for those who sleep enough or consumed low amounts of alcohol before going to bed.

Hence, we tested in a number of post-hoc analyses whether the relationship between pre-vacation and during-vacation sleep quality varied as a function of the number of hours sleep and of alcohol consumption before going to sleep during vacation (i.e. the number of glasses of alcoholic beverages). These analyses revealed no main or moderator effects of sleep hours $\left(F_{\mathrm{S}}(1,92)<1.26, n s\right)$ on sleep quality. The same was true for alcohol consumption $\left(F_{\mathrm{S}}(1,92)<1.20, n s\right)$. So we concluded that neither the number of hours the participants slept, nor alcohol consumption during vacation explained why sleep quality did not improve during vacation. We cannot rule out that physical sleeping circumstances may have accounted for the absence of a vacation effect on sleep quality.

Contrary to our expectations, we found the lowest levels of fatigue immediately after vacation instead of during vacation. Strictly speaking, this effect cannot be labelled an after-effect of vacation, since levels of fatigue on vacation did not differ significantly from pre-vacation levels, indicating the absence of a vacation effect. Still, we assume that decreased levels of fatigue on post-vacation may represent a vacation after-effect: during winter sports vacation, people engage in physically demanding, uncommon activities which are presumably accompanied by feeling physically fatigued, while after work, people may feel primarily mentally fatigued.

\section{Vacation after-effects}

The results regarding vacation after-effects were less favourable for health and wellbeing: the five positive vacation effects had vanished within the first week of workresumption. Fatigue constituted the only exception to this rule and was lowest immediately after vacation. Despite the absence of a vacation effect in fatigue, this finding is in line with the slower fade-out process in burnout that Westman and Eden (1997) reported and may point to positive mid-term effects regarding fatigue.

Due to the absence of on-vacation measurement occasions, most previous vacation studies defined a vacation effect as the difference between the pre-vacation and post-vacation levels in health and well-being that "sandwiched" the vacation period. Whereas the meta-analysis of De Bloom et al. (2009) revealed a small shortterm after-effect, we found none in the current study. There are several possible explanations for the immediate fade-out of vacation effects that need to be discussed.

Could it be that the type of vacation is important for the duration of the vacation effects? One might argue that a winter sports vacation as a very active type of vacation may have less enduring beneficial health effects than, for example, a predominantly relaxing vacation. However, research has demonstrated that active leisure activities, in particular physical activities, improve well-being and may be even more recovering than low-effort activities like watching television (Rook \& Zijlstra, 2006; Sonnentag, 2001; Sonnentag \& Natter, 2004;). Accordingly, it is not very likely that the active character of a winter sports vacation explains the lack of after-effects. 
Another explanation may be that a winter sports vacation normally forms an interruption of a busy period of the year. Vacationers return home and are immediately trapped in demanding daily routines and hassles like unpacking and washing clothes, work and non-work-obligations. Research on spa therapy suggests that returning home in the second half of a workweek with the weekend in prospect is more favourable for the conservation of positive effects than returning on Sunday with a full working week ahead (Strauss-Blasche, Muhry, Lehofer, Moser, \& Marktl, 2004). Therefore, it would be interesting to examine in future studies whether short vacations (active or passive) scheduled at a more relaxed time of the year (e.g. during a long summer vacation) or in a different manner (e.g. one or two more days off after returning home to prevent "post-vacation stress") may have more enduring aftereffects.

Third, the duration of the vacation period may constitute a major component of its effectiveness in improving health and well-being and its after-effects. Just as a lower dose of medicine may be less effective in curing a disease, a short vacation may have fewer and less profound effects on health and well-being than a long vacation period. A winter sports vacation is typically a short vacation type: most of our participants spent only nine days away from home (including two travel days) and one week away from work. As a consequence of the brief "treatment," the effects may have been weaker and more short-lived.

It may also be that in previous studies the after-effects of vacation have been overestimated. If the pre-vacation occasion is programmed immediately before vacation, it may be confounded by preparation stress for the vacation which is likely to be associated with decreased levels of health and well-being. When this prevacation occasion is subsequently treated as baseline, vacation after-effects would artificially increase.

Regarding the rapid fade-out process of positive vacation effects, an intriguing question may be: Why should we go on vacation at all when effects wash out so fast? However, like any other freely chosen and pleasant activity, a vacation is a period that people enjoy for its own sake; vacation makes people happy and healthy as our study unmistakably showed. A vacation is, therefore, an effective, strong and natural way to boost the well-being of employees.

Furthermore, health and well-being could deteriorate over time if people did not go on vacation, as vacation is important for long-term health and vitality, and for building up enduring personal resources and coping capacities. A study of Gump and Matthews (2000), for example, showed that not taking annual vacations was associated with a higher risk of mortality during a nine-year period. In our view, a more appropriate question regarding the temporal nature of vacation effects would correspondingly be: Is it possible to conserve positive vacation effects, and if so, which strategies can be used to slow down fade-out processes and prolong vacation relief (see also Eden, 2001)?

\section{Limitations}

The limited variation in vacation type and duration was a deliberate choice in the current study. The uniformity with respect to activities, duration and time off the job before and after vacation (maximally one or two days) enabled us to generate reliable results for short winter sports vacations. However, the question remains whether we 
would have found the same pattern of results for other vacation types, for other vacations durations and for other periods (seasons) of the year.

In addition, our sample of skiing enthusiasts may limit the external validity of our study. Although our sample was heterogeneous in many regards (gender, age, type of work, family background), winter sports vacationers may be above-average healthy, active and sporty. Even though we do not have theoretical reasons to assume that vacations will have less positive effects among less healthy and sporty individuals, we should be careful in generalizing our findings.

Another limitation is the use of self-reports only. However, health and well-being are by definition subjective constructs and self-reports are probably the best way to measure them (Kompier, 2005). But one may also argue that retrospective evening scores may be biased by cognitive distortions like the "rosy view bias." Mitchell, Thompson, Peterson, and Cronk (1997) found that people's post-event recollections are more positive than their evaluations of the actual experiences. Yet, we reduced such potential biases by measuring several times a week and by asking respondents to indicate their level of health and well-being on the same day.

We measured fatigue with a single-item measure because it reduced the burden put on the participants, prevented non-response and attrition and because it is a valid substitute for multiple item measures of fatigue (Van Hooff et al., 2007). In spite of that, the use of two additional single-item measures on mental and physical fatigue could have provided more in-depth information and understanding of the vacation (after-) effects of fatigue.

Finally, there may be an effect of the time of the day at which the pre- and postvacation measures (just before going to bed) and the on-vacation measures (between 5 and $7 \mathrm{pm}$ ) were taken. It may be that people feel better in the early evening than just before going to bed because of feeling more tired at bedtime. Nevertheless, fatigue was highest during vacation, in the early evening, which does not point into the direction of a "before bedtime effect."

\section{Suggestions for future vacation research}

First and foremost, future vacation research could be optimized by applying research designs like the one we used with repeated measures pre-, inter- and post-vacation. Furthermore, the combination of different technically innovative instruments for data collection (digital diaries, telephone surveys) and an extensive protocol to guarantee compliance (careful recruitment, SMS reminders) may help future researchers to start measuring on vacation and to prevent attrition.

Data triangulation, for example, the combination of self-reports, ratings from the partner or fellow vacationers and performance ratings, would be a means to further improve vacation research and to generate valid and reliable results.

Some other suggestions for future vacation research regarding sleep quality (i.e. take physical sleep circumstances into account) and fatigue (i.e. distinguish mental and physical fatigue) are important and were already briefly mentioned above.

Because different types of vacation (active and passive) may have different effects on health and well-being, the impact of various vacation types on the strengths and duration of vacation effects should be investigated (see also Eden, 2001). For instance, would a relatively short relaxing vacation during the winter period have the 
same vacation effects and (lack of) after-effects as an active winter sports vacation? Also, the impact of similar types of vacation (e.g. physically active vacations) scheduled in different seasons of the year could be examined. Would, for instance, an active vacation in the summer (e.g. sailing or biking in the summer holidays) have the same vacation and after-effects as an active vacation in the winter?

The role of vacation duration is difficult to study because when duration varies a lot of other variables such as vacation type and activities co-vary. As a consequence, it will be impossible to attribute vacation effects and after-effects mainly to its duration. It does for example not make sense to compare vacation effects of a fourweek backpacker-trip through Scandinavia with a two-week all-inclusive resort stay at Costa del Sol. Also experimentally, assigning participants to different vacation durations is practically impossible (for creative ideas like give-away paid vacations see Eden, 1990). So, the best way to study the effects of vacation duration is probably to vary vacation duration while holding vacation type as constant as possible.

Another interesting research topic is the investigation of the role of work accumulation as moderator of vacation (after-) effects. For some employees work may pile up before vacation (see also DeFrank, et al., 2000; Westman, 2004, 2005); they have to work harder in order to go on vacation and experience "working-ahead stress." On vacation, their work may accumulate even further and they may be confronted with high workload after returning home (Fritz \& Sonnentag, 2006). We may call this "catch-up stress." For other employees, work may be structured in a different way and may not pile up because a colleague takes over. Accordingly, it would be interesting to include measures of "working ahead-stress" before and "catch-up stress" after vacation and study their effects on health and well-being.

A target for vacation researchers could also be the investigation of the role of vacation activities and experiences on health and well-being. Up till now, vacation remains an intervention with more or less unknown content and we do not know if vacation activities like physical activities, relaxing, household or work-related tasks have a different impact on the strength of the vacation effect or the fade-out rate (for an exception see Fritz \& Sonnentag, 2006). Vacation expectations and their fulfilment, uplifts and hassles and relations with travel companions and the life partner during vacation are additional examples for possible moderators of the vacation effect which should be studied (see also Eden, 2001).

Last but not least, strategies to slow down fade-out processes and to prolong vacation relief are an important avenue for future research. Positive, frequent vacation reflection may be a prime candidate for fade-out deceleration because reflecting repeatedly and favourably on pleasant vacation experiences may reactivate positive vacation cognitions and feelings and enhance health and well-being. In an experiment on cardiovascular reactivity (Fredrickson, Mancuso, Branigan, \& Tugade, 2000), positive emotions speeded up cardiovascular recovery from stress, indicating that positive emotions regulate or even undo negative emotional arousal. These findings support the assumption from Broaden-and-Build Theory (Frederickson, 2001) that positive emotions may improve individual's coping capacity to deal with stressors. So, positive emotions experienced during vacation and positive vacation reflection may protect and build resources that improve health and wellbeing by buffering future threats.

In conclusion, it seems that a winter sports vacation certainly improves health and well-being, but positive effects are short-lived. Future vacation studies should 
therefore focus on means to decelerate the fade-out process in order to prolong vacation relief. Moreover, we propose a longitudinal framework for vacation research with proper baseline-, on-vacation- and multiple post-vacation measurements (such as in the framework that we employed) to investigate the effects of different vacation types, durations, activities and experiences on health and wellbeing in future vacation studies.

\section{Acknowledgements}

We thank Lineke Berendsen for her help in collecting the data for this study and Pieter van Groenestijn for helping digitalizing the diaries and the preparation of the data set. Our special thanks also go to the two reviewers for their valuable, competent comments to improve our manuscript.

\section{References}

Akerstedt, T. (2006). Psychosocial stress and impaired sleep. Scandinavian Journal of Work, Environment \& Health, 32, 493-501.

Belkic, K., Landbergis, P.A, Schnall, P.L., \& Baker, D. (2004). Is job strain a major source of cardiovascular disease risk? Scandinavian Journal of Work, Environment \& Health, 30, $85-128$.

Brosschot, J.F., Van Dijk, E., \& Thayer, J.F. (2007). Daily worry is related to low heart rate variability during waking and the subsequent nocturnal sleep period. International Journal of Psychophysiology, 63, 39-47.

Cohen, J. (1988). Statistical power analysis for the behavioral sciences. Hillsdale, NJ: Lawrence Erlbaum.

Dahlgren, A., Kecklund, G., \& Akerstedt, T. (2005). Different levels of work related stress and the effects on sleep, fatigue and cortisol. Scandinavian Journal of Work, Environment \& Health, 31, 277-285.

De Bloom, J., Kompier, M., Geurts, S., De Weerth, C., Taris, T., \& Sonnentag, S. (2009). Do we recover from vacation? Meta analysis of vacation effects on health and well being. Journal of Occupational Health, 51, 13-25.

DeFrank, R.S., Konopaske, R., \& Ivancevich, J.M. (2000). Executive travel stress: Perils of the road warrior. The Academy of Management Executive, 14, 58-71.

De Lange, A.H., Taris, T.W., Kompier, M.A.J., Houtman, I.L.D., \& Bongers, P.M. (2003). The very best of the millennium: Longitudinal research and the demand control (support) model. Journal of Occupational Health Psychology, 8, 282-305.

DeShon, R.P., \& Morris, S.B. (2003). Modeling complex data structures: The general linear model and beyond. In S.G. Rogelberg (Ed.), Handbook of research methods in industrial and organizational psychology (pp. 390-411). Malden, MA: Blackwell.

Eden, D. (1990). Acute and chronic job stress, strain, and vacation relief. Organizational Behavior and Human Decision Processes, 45, 175-199.

Eden, D. (2001). Vacations and other respites: Studying stress on and off the job. In C. Cooper \& I.T. Robertson (Eds.), Well being in organizations (pp. 305-330). West Sussex, UK: John Wiley.

Elo, A., Leppänen, A., \& Jahkola, A. (2003). Validity of a single item measure of stress symptoms. Scandinavian Journal of Work, Environment \& Health, 29, 444-451.

Etzion, D. (2003). Annual vacation: Duration of relief from job stressors and burnout. Anxiety, Stress and Coping, 16(2), 213-226.

Ferrie, J.E., Westerlund, H., Virtanen, M., Vahtera, J., \& Kivimaki, M. (2008). Flexible labour markets and employee health. Scandinavian Journal of Work, Environment \& Health, 6, $98-110$. 
Frederickson, B.L. (2001). The role of positive emotions in positive psychology: The broaden and build theory of positive emotions. American Psychologist, 56, 218-226.

Fredrickson, B.L., Mancuso, R.A., Branigan, C., \& Tugade, M.M. (2000). The undoing effect of positive emotions. Motivation and Emotion, 24, 237-258.

Fritz, C., \& Sonnentag, S. (2005). Recovery, health, and job performance: Effects of weekend experiences. Journal of Occupational Health Psychology, 10, 187-199.

Fritz, C., \& Sonnentag, S. (2006). Recovery, well being, and performance related outcomes: The role of workload and vacation experiences. Journal of Applied Psychology, 91, 936-945.

Geurts, S.A.E., \& Sonnentag, S. (2006). Recovery as an explanatory mechanism in the relation between acute stress reactions and chronic health impairment. Scandinavian Journal of Work, Environment \& Health, 32, 482-492.

Gump, B.B., \& Matthews, K.A. (2000). Are vacations good for your health? The 9 year mortality experience after the multiple risk factor intervention trial. Psychosomatic Medicine, 62, 608-612.

Hjortskov, N., Rissen, D., Blangsted, A.K., Fallentin, N., Lundberg, U., \& Sogaard, K. (2004). The effect of mental stress on heart rate variability and blood pressure during computer work. European Journal of Applied Physiology, 92, 84-89.

Kompier, M. (2005). Assessing the psychosocial work environment - "subjective" versus "objective" measurement. Scandinavian Journal of Work, Environment \& Health, 31, 405-408.

McEwen, B.S. (1998). Stress, adaptation, and disease: Allostatis and allostatic load. Annals of the New York Academy of Science, 840, 33-44.

Meijman, T.F., \& Mulder, G. (1998). Psychological aspects of workload. In P.J.D. Drenth, H. Thierry, \& C.J. de Wolff (Eds.), Handbook of work and organizational psychology. Work psychology (2nd ed., Vol. 2, pp. 5-33). Hove: Psychology Press.

Meyers, A.R., Perrine, M.W., \& Caetano, R. (1997). Alcohol use and downhill ski injuries: A pilot study. In R.J. Johnson, C.D. Mote, Jr., \& A. Ekeland (Eds.), Skiing trauma and safety (Vol. 11, pp. 14-22). West Conshohocken, PA: American Society for Testing and Materials.

Mitchell, T.R., Thompson, L., Peterson, E., \& Cronk, R. (1997). Temporal adjustment in the evaluation of events: The "Rosy View". Journal of Experimental Social Psychology, 33, 421-448.

Roehrs, T., \& Roth, T. (2001). Sleep, sleepiness, sleep disorders and alcohol use and abuse. Sleep Medicine Reviews, 5, 287-297.

Rook, J.W., \& Zijlstra, F.R.H. (2006). The contribution of various types of activities to recovery. European Journal of Work and Organizational Psychology, 15, 218-240.

Sluiter, J.K., Frings Dresen, M.H., Meijman, T.F., \& Van der Beek, A.J. (2000). Reactivity and recovery from different types of work measured by catecholamines and cortisol: A systematic literature review. Occupational Environmental Medicine, 57, 289-315.

Sonnentag, S. (2001). Work, recovery activities, and individual well being: A diary study. Journal of Occupational Health Psychology, 6, 196-210.

Sonnentag, S., \& Natter, E. (2004). Flight attendants' daily recovery from work: Is there no place like home? International Journal of Stress Management, 11, 366-391.

Strauss Blasche, G., Muhry, F., Lehofer, M., Moser, M., \& Marktl, W. (2004). Time course of well being after a three week resort based respite from occupational and domestic demands: Carry over, contrast and situation effects. Journal of Leisure Research, 36, 293-309.

Taris, T.W. (2000). A primer in longitudinal data analysis. London: Sage.

Van Hooff, M.L.M., Geurts, S.A.E., Kompier, M.A.J., \& Taris, T.W. (2007). Workdays, in between workdays, and the weekend: A diary study on effort and recovery. International Archives of Occupational and Environmental Health, 80, 599-613.

Van Hooff, M.L.M., Geurts, S.A.E., Kompier, M.A.J., Taris, T.W., Houtman, I.L.D., \& Van den Heuvel, F. (2005). Disentangling the causal relationships between work home interference and employee health. Scandinavian Journal of Work, Environment \& Health, $31,15-29$. 
Van Hooff, M.L.M., Geurts, S.A.E., Taris, T.W., \& Kompier, M.A.J. (2007). "How fatigued do you currently feel?" Convergent and discriminant validity of a single item fatigue measure. Journal of Occupational Health, 49, 224-234.

Westman, M. (2004). Strategies for coping with business trips: A qualitative exploratory study. International Journal of Stress Management, 11(2), 167-176.

Westman, M. (2005). The impact of short business travels on the individual, the family and the organization. In A. Antonio \& C. Cooper (Eds.), Research companion to organizational health psychology (pp. 478-491). Cheltenham, England: Edward Elgar.

Westman, M., \& Eden, D. (1997). Effects of a respite from work on burnout: Vacation relief and fade out. Journal of Applied Psychology, 82, 516-527.

Westman, M., \& Etzion, D. (2001). The impact of vacation and job stress on burnout and absenteeism. Psychology \& Health, 16(5), 595-606. 